\title{
Rosai-Dorfman disease: A rare clinico-pathological presentation
}

\author{
Bhushan M. Warpe, ${ }^{1}$ Shraddha V. More ${ }^{2}$
}

1.Department of Pathology, Indira Gandhi Government Medical College, Nagpur, India

2. Department of Biochemistry, Swami Ramanand Teerth Rural Medical College, Ambajogai,India

\section{CASE REPORT}

Please site this paper as: Warpe BM, More SV. RosaiDorfman disease: $\mathrm{A}$ rare clinico-pathological presentation. AMJ 2013. AMJ 2014, 7, 2, 68-72. http://doi.org/10.21767/AMJ.2014.1931

\section{Corresponding Author:}

Bhushan M. Warpe

Room no. 89, NRH, IGGMC, Nagpur city,

Pincode-440018, Maharashtra state, India.

Contact: +917303134083

Email: bhushan.warpe@gmail.com

\section{Abstract}

Rosai-Dorfman disease (RDD) is also known as Sinus Histiocytosis with Massive Lymphadenopathy (SHML). It is a rare, benign, self-limiting disease of phagocytic histiocytes affecting a young age group presenting with massive painless cervical lymphadenopathy. RDD has nodal and also extra-nodal involvement with episodes of exacerbation and remissions, whose aetiology remains poorly elucidated. It is highly variable in its clinical presentation and response to treatment. Its treatment is poorly defined but the prognosis is usually favourable. Here we are reporting a rare, unusual clinical presentation of cervical lymphadenopathy and nasal mass diagnosed as RDD with cyto-histopathological correlation. Only a few such cases have been reported in the literature. We also emphasise that clinicians and pathologists should always be aware of RDD in making a differential diagnosis of cervical lymphadenopathy.

\section{Key Words}

Rosai-Dorfman Disease, cervical lymphadenopathy, histiocytes, emperipolesis

\section{What this study adds:}

\section{What is known about this subject?}

Rosai-Dorfman disease (RDD) is a rare, benign disease that presents with massive painless cervical lymphadenopathy. It affects younger people.

\section{What is the key finding in this case report?}

This case is an unusual and rare clinical presentation of cervical lymphadenopathy and nasal mass diagnosed as RDD with cyto-histopathological correlation.

\section{What are the implications for future practice?}

Clinicians and pathologists should be aware of RDD in making a differential diagnosis of cervical lymphadenopathy.

\section{Background}

In 1969, Rosai and Dorfman first described a newly recognised benign systemic histioproliferative disease characterised clinically by bilateral painless cervical lymphadenopathy, fever, leucocytosis, and pathologically by pericapsular fibrosis with dilated sinuses, large histiocytes with intact phagocytosed lymphocytes (emperipolesis), heavily infiltrated with large histiocytes, lymphocytes and plasma-cells in the background. They named the entity "sinus histiocytosis with massive lymphadenopathy" (SHML or Rosai-Dorfman disease). ${ }^{1}$

The head and neck region is the preferred site of the extranodal form of RDD. Extranodal involvement is seen in 40 per cent of RDD cases, which show similar morphological features to its nodal counterpart, although fewer histiocytes with emperipolesis are encountered. ${ }^{2}$ Literature reviews show that up until 2004 about 600 cases of RDD had been reported, in all races but mainly in Caucasians (43 per cent), in any age group, but mainly in first and second decades (81 per cent), and more in males than females $(2: 1){ }^{3}$ The most common presenting symptom of this disease is painless bilateral cervical lymphadenopathy which is seen in 90 per cent of the patients, and in 43 per cent of the cases the patients have at least one site of extra-nodal involvement. ${ }^{4,5}$ Rarely sites other than lymph nodes are involved such as 
skin and soft tissue, upper respiratory system, genitourinary tract, eye, orbit, kidney, thyroid, breast, or bone. ${ }^{6}$

Rosai-Dorfman disease is rare, especially in the Indian subcontinent where only two cases have been reported with extra-nodal, sino-nasal mucosal involvement. ${ }^{4,5}$ It is reported that around 40 per cent cases of RDD can have extra-nodal involvement. Both physicians and pathologists must be aware of rare disorders like RDD, which manifest as lympho-proliferative disorders with generalised lymphadenopathy. Here, we discuss a paediatric RDD case with cervical lymphadenopathy associated with nasal mass. ${ }^{6}$

\section{Case details}

A 12-year-old male came with complaints of painless bilateral neck swelling with bilateral nasal obstruction and nasal bleed since one year. Swelling was insidious at onset and progressive in nature. Nasal obstruction was also insidious at onset and progressed over a period of time with intermittent episodes of spontaneous nasal bleed, which stopped on its own without any medication. There was no history of recurrent throat pain, difficulty in swallowing, loosening of clothes, decreased appetite, or fever.

\section{Figure 1: Clinical photograph-Enlarged left cervical lymph} node (level III).

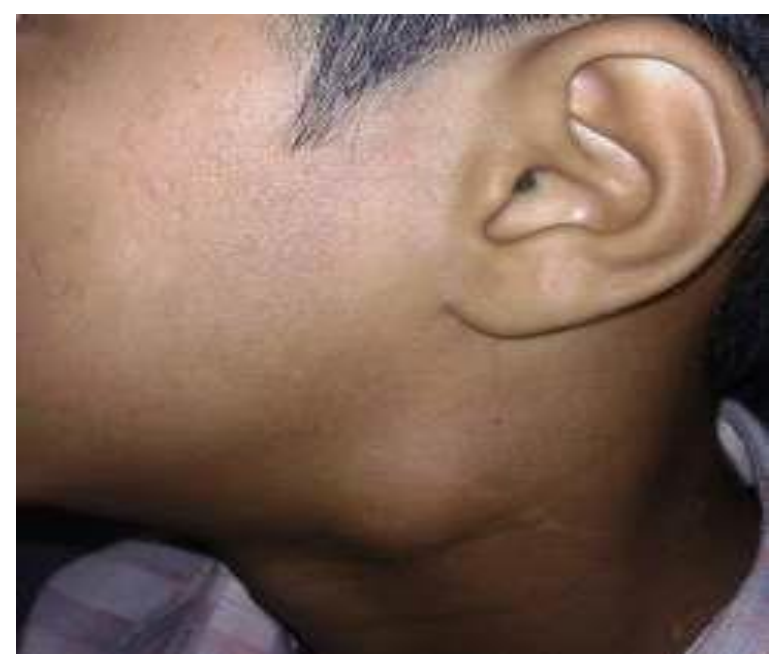

On local examination, $3 \times 3 \mathrm{~cm}$ swelling was present bilaterally in the level III lymph nodal region. It was firm, non-tender, non-fluctuant, and mobile (Figure 1). Bilateral nasal mass presented anteriorly, partially obstructing the anterior nasal nares (Figure 2).
Figure 2: Clinical photograph-Bilateral anterior nasal mass partially obstructing the anterior nasal nares

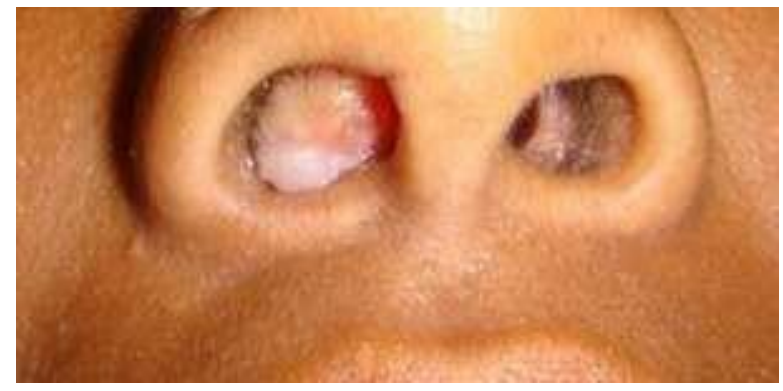

USG and CT scan of the neck were suggestive of bilateral cervical lymphadenopathy in level III without any caseation or matting. Anterior and posterior rhinoscopy was suggestive of bilateral sinonasal polyps. The $\mathrm{CBC}$ of the patient revealed $\mathrm{Hb}-12.0 \mathrm{gm} / \mathrm{dl}$. TLC-9400 cells/cmm, DLC: $\mathrm{P}-68 \%, \mathrm{~L}-28 \%, \mathrm{M}-02 \%, \mathrm{E}-02 \%$ and platelets $-3.15 \mathrm{lacs} / \mathrm{cm}$. The ESR of the patient was $13 \mathrm{~mm}$ at the end of 1 hour by Westergren method, which was within normal limits considering the patient's age. The RFTs, LFTs, serum albumin, and serum globulin were within normal limits. HIV and tuberculin tests were negative. Non-contrast CT of paranasal sinuses showed polypoidal soft-tissue mass within bilateral nasal cavities, along bilateral middle and left inferior turbinates.

Figure 3: Microphotograph-FNAC smear showing large histiocytes with 'emperipolesis'. (MGG, x 400)

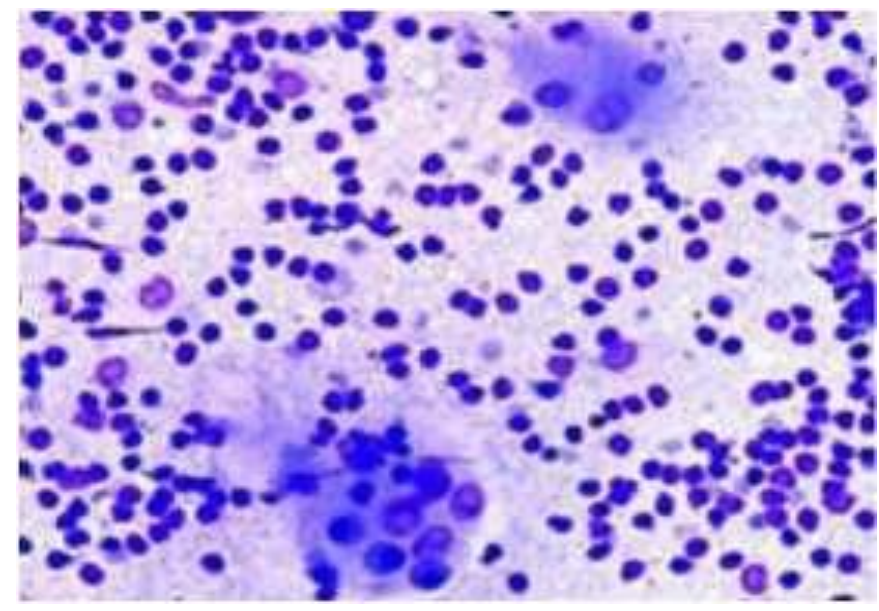

The Fine Needle Aspiration Cytology (FNAC) of the cervical lymph nodes in this case was performed from multiple sites and blood-stained aspirates were obtained. Smears were stained with MGG, PAP, and H\&E stains. On microscopic examination, cytological smears revealed the presence of diffusely distributed histiocytes throughout the FNAC smears, pale cytoplasm with single to multilobated or multiple nuclei but no nuclear atypia. The cytoplasm of these histiocytes exhibited numerous intact lymphocytes (emperipolesis) as well as the presence of neutrophils. In some histiocytes, the phagocytosed cells were so numerous 
that they obscured the nucleus. The nuclei showed fine chromatin and inconspicuous to prominent nucleoli. The background had mature lymphocytes, plasma cells, neutrophils, and tingible body macrophages. Based on these characteristic cytological findings, a diagnosis of RosaiDorfman disease was made (Figure 3). Tuberculosis was ruled out as a clinical suspicion as there was no caseation necrosis, epitheloid cells, or Langhans giant cells with noncontributory AFB smears.

Figure 4: Microphotograph-Tissue section from cervical lymph node showing partial effacement of its architecture with prominent dilated lymph sinuses. (H\&E, x40)

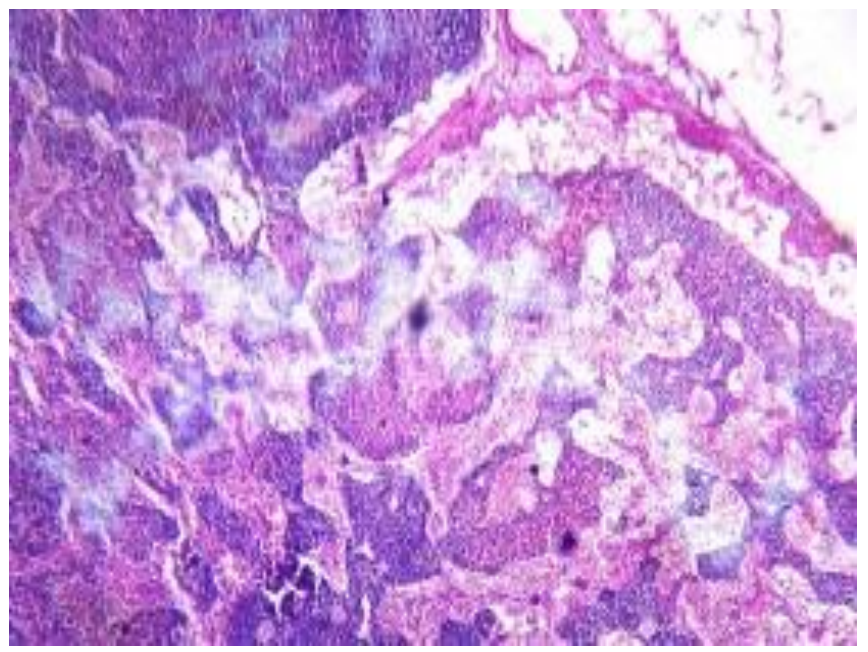

Subsequently, for confirmation of the same, a biopsy of the cervical lymph node was done. Microscopic examination of the sections, cut from paraffin-embedded blocks, showed fibrous thickening of the lymph node capsule and prominent dilatation of lymph sinuses resulting in partial architecture effacement (Figure 4). The primary lymphoid follicles were present mostly at the cortical areas. The sinuses were occupied by numerous histiocytes having abundant pale cytoplasm and phagocytosed lymphocytes (emperipolesis), plasma cells and occasional neutrophils (Figure 5). Thus, the cervical lymph node biopsy was in favour of RDD on histopathology.
Figure 5: Microphotograph-Tissue section from cervical lymph node showing large histiocytes with 'emperipolesis' in lymph sinuses. (H\&E, x 400)

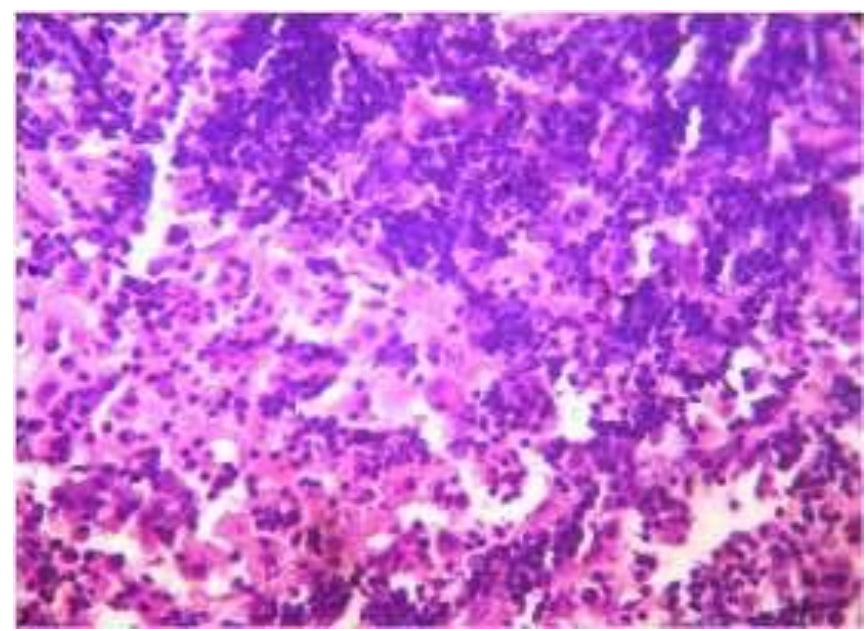

The case had an extra-nodal involvement in the form of a nasal mass, which on biopsy showed features of ulcerated respiratory lining of the nasal mucosa (Figure 6). The underneath sub-epithelial tissue revealed sinus histiocytosis with few histiocytes showing characteristic emperipolesis. This simulated the associated histological features that were seen in cervical lymph node biopsy.

Figure 6: Microphotograph-Tissue section showing ulcerated nasal mucosa with lymphoplasmacytic infiltrate and few histiocytes with 'emperipolesis' in sub-epithelial tissue. (H\&E, x 400)

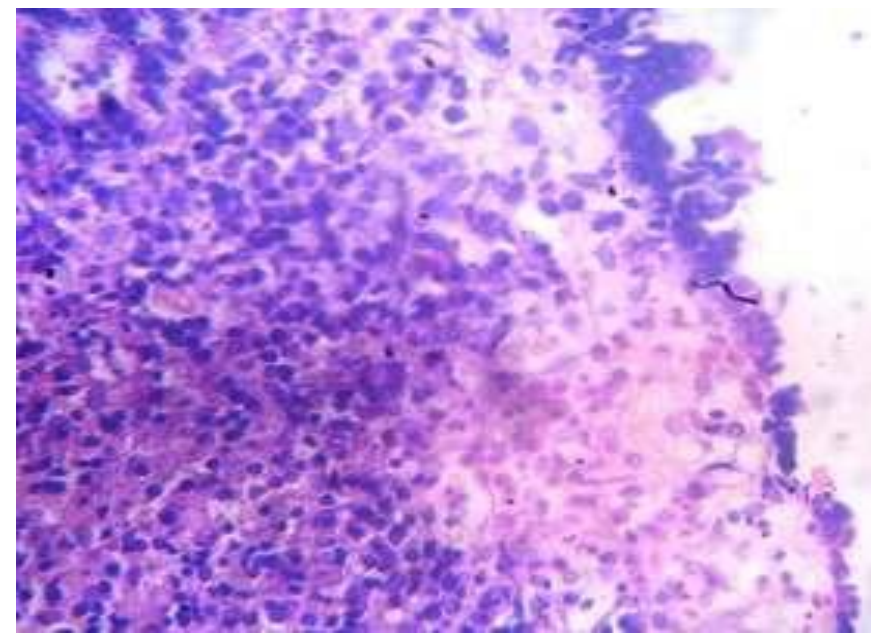

Thus the FNAC diagnosis of RDD in the cervical lymph node smears were confirmed on biopsy. Also, the tissue sections from the biopsied nasal mass were reported as RDD. Due to financial constraints and our laboratory set-up, immunohistochemistry, that is, histiocytic positivity for S-100 protein and $C D-68$ protein, which is now considered the gold standard, could not be performed in our case. But fineneedle aspiration cytology and biopsy play an important diagnostic role in SHML or RDD and may be conclusive in a 
typical clinical setting. So, in our case, a RDD diagnosis was established based on the biopsy report and the typical clinical setting.

The diagnosis of RDD is made on the basis of clinical suspicion, supported by cytology, and confirmed by histopathology. The head and neck region is the preferred site of the extra nodal form of the disease. Mostly, the lymph nodes regress on their own; however, corticosteroids are given to the patients with progressive RDD. In our case, an oral tablet of prednisolone $(0.5 \mathrm{mg} / \mathrm{kg}$ of patient's weight) was started. The patient responded very well to treatment over a period of two weeks; his nasal mass subsided within that time. Subsequently, the patient was given tapered doses of oral prednisolone over the next two weeks and asked to follow up. There was no evidence of recurrence on follow-up over a period of eight months in our RDD case.

\section{Discussion}

In our case, the child was 12 years old, whereas the two reported Indian studies were adults. ${ }^{3,7}$ Also, unlike in the other two cases, in this case the haematological parameters and ESR were within normal limits and there was no associated fever.

In an Indian study by Mehrotra et al., ${ }^{8}$ the RDD patient had multiple lymph node swellings of various sites involved. This patient responded to an anti-chemotherapeutic trial. Our case had only cervical lymph nodes involved with extranodal, nasal mass and as the patient was poor, S-100 could not be done. Our patient responded well to the therapeutic trial of the oral steroid drug (prednisolone) rather than the costlier chemotherapeutic trial by Mehrotra et al. ${ }^{8}$

SHML or RDD is a rare but well-defined, histiocytic proliferative disorder of unknown aetiology characterised frequently by spontaneous remission. Some investigators consider it to be of bone marrow stem cell origin. More than 90 per cent of patients with SHML present with massive bilateral mobile and non-tender cervical lymphadenopathy. These nodes may at times become matted and prominent due to pericapsular fibrosis. Lowgrade fever is generally present along with, normocytic normochromic anaemia, elevated ESR, leucocytosis, and hyperglobulinaemia, which are non-specific clinical findings. ${ }^{9}$ In our case, there was no associated fever with bilateral cervical lymphadenopathy, nor was there any associated anaemia, elevated ESR, leucocytosis, or hyperglobulinaemia.
The aetiology of RDD is unclear. RDD may be either familial or infection-induced. It has been suggested to result from an immune response to an infectious agent or an aberrant response of macrophages to cytokines. However, no single antibacterial or antiviral agent has been associated. Infectious agents that have been studied in association with RDD include Epstein Barr Virus (EBV), and Human Herpes Virus 6 (HHV-6). ${ }^{10}$

A familial association has been observed in some cases. ${ }^{10}$ It has been found that stimulation of monocytes/macrophages via macrophage colony stimulating factor (M-CSF) lead to their transformation into numerous large histiocytes with abundant, pale cytoplasm. Some macrophages even phagocytise lymphocytes, which is called "emperipolesis". Lymphophagocytosis (emperipolesis) is thus of great diagnostic significance for RDD. The background typically shows lymphocytes, plasma cells, and occasional neutrophils. ${ }^{2,11}$

Extra nodal involvement is seen in 40 per cent of RDD cases which show similar morphological features to its nodal counterpart, although fewer histiocytes with emperipolesis are encountered. ${ }^{2,12}$ The internalised lymphocytes are usually located within cytoplasmic vacuoles.

Ultrastructurally, histiocytes in RDD lack cytoplasmic Birbeck granules unlike in Langerhans cell histiocytosis. On immunostaining RDD show positivity for S-100 protein, CD11c, CD14, CD33, and CD68 antigens and are CD1a negative. ${ }^{9}$ Only two cases of RDD progression, one to malignant lymphoma and another to amyloidosis have been documented. $^{9}$ The treatment modalities for RDD are nonspecific and include corticosteroids, chemotherapy, antibiotic therapy, radiation therapy, and surgical treatment. ${ }^{13}$ Complications are mostly due to pressure effects exerted by the enlarged cervical lymph nodes. So, surgical procedures are mainly done to relive pressure symptoms, if any.

A careful interpretation of the morphology of the histiocytes on FNAC is required particularly, when they are few in number, as the diagnosis may be missed at the initial stages of the disease. The FNAC findings of SHML should always be clinically correlated to give a diagnosis of RDD. FNAC can be used as a reliable tool to establish a diagnosis and an unnecessary biopsy which is an invasive procedure can be avoided. In view of need to keep a follow-up, cytology offers a useful tool, especially because RDD has a propensity to recur. If there is an extra nodal clinical manifestation, like in our RDD case, where lymph node aspirate shows a possibility of SHML on cytology, then a 
FNAC or biopsy should be performed on the extra nodal site and the findings of both the sites can be correlated. Also, a simple therapeutic dose of oral corticosteroid-prednisolone helps in managing RDD.

Thus this case report aims at creating knowledge of this rare entity because clinicians, general practitioners and pathologists should always be aware of RDD in making a differential diagnosis of cervical lymphadenopathy.

\section{References}

1. Rosai J, Dorfman RF. Sinus histiocytosis with massive lymphadenopathy. Arch Pathol 1969;87(1):63-70.

2. Sennes L, Koishi H, Cahali R, Sperandio F, Butugan $O$. Rosai Dorfman disease with extranodal manifestation in the head. Ear Nose Throat J 2004;83 (12):844-7.

3. Bist SS, Bisht M, Varshney S, Pathak VP. Rosai Dorfman Syndrome with Extranodal Manifestation. J Assoc Physicians India. 2007;55:445-7.

4. Sujata G. Multifocal, Extranodal Sinus Histiocytosis With Massive Lymphadenopathy. An Overview. Arch Pathol Lab Med 2007;131(7):1117-21.

5. Sachdev R, Setia N, Jain S. Sinus histiocytosis with massive lymphadenopathy. Is the lymph node enlargement always massive? Med Oral Patol Oral Cir Buccal 2007;12(3):198-200.

6. Ju J1, Kwon YS, Jo KJ, Chae DR, Lim JH, Ban HJ et al. Sinus histiocytosis with massive lymphadenopathy: a case report with pleural effusion and cervical lymphadenopathy. J Korean Med Sci 2009;24(4):760-2

7. Agarwal P, Prakash P, Handa A, Gupta AK. Rosai-Dorfman disease with sinonasal mucosal involvement. JIACM 2012;13(3): 225-8.

8. Mehrotra S, Ather S, Gupta P, Mehrotra B. Rosai-Dorfman disease - A Clinicopathological Presentation. JAPI 2007; 55: 587-9.

9. Rosai J, Dorfman RF. Sinus histiocytosis with massive lymphadenopathy: A pseudolymphomatous benign disorder. Analysis of 34 cases. Cancer 1972;30(5):1174-88.

10. Harley EH. Sinus histiocytosis with massive lymphadenopathy (Rosai-Dorfman disease) in a patient with elevated Epstein-Barr virus titer. J Natl Med Assoc 1991;83(10):922-4.

11. Jani PA, Banjan D. A case of Sinus Histiocytosis with Massive Lymphadenopathy (Rosai- Dorfman Syndrome ) from Western India. Mcgill J Med 2008;11(2):156-9.

12. Li S, Yan Z, Zhala N, Zhala Q. Fine needle aspiration diagnosis of Rosai Dorfman disease in an osteolyic lesion. CytoJournal 2010;7:12.

13. Kushwaha R, Ahluwalia C, Sipayya V. Diagnosis of Sinus Histiocytosis with massive lymphadenopathy (Rosai-
Dorfman Disease) by fine needle aspiration cytology. J Cytol 2009;26(2):83-5.

\section{ACKNOWLEDGEMENTS}

Department of ENT, IGGMC, Nagpur, India.

\section{PEER REVIEW}

Not commissioned. Externally peer reviewed.

\section{CONFLICTS OF INTEREST}

The authors declare that they have no competing interests.

\section{PATIENT CONSENT}

The authors declare that:

1. They have obtained written, informed consent for the publication of the details relating to the patient(s) in this report.

2. All possible steps have been taken to safeguard the identity of the patient(s).

3. This submission is compliant with the requirements of local research ethics committees. 